Psicologia Escolar

e Educacional
ARTIGO

DOI: http://dx.doi.org//10.1590/2175-35392021226072

Localizador - e226072

\title{
ADAPTAÇÃO ACADÊMICA À UNIVERSIDADE: RELAÇÕES ENTRE MOTIVAÇÃO, EXPECTATIVAS E HABILIDADES SOCIAIS
}

\author{
Adriana Benevides Soares ${ }^{1,2} \mathbb{D}$; Marcia Cristina Monteiro ${ }^{2} \mathbb{D}$; Humberto Claudio Passeri Medeiros ${ }^{2} \mathbb{D}$; Fátima de \\ Almeida Maia ${ }^{2} \mathbb{D}$; Roberta de Souza Nogueira Barros ${ }^{2} \mathbb{D}$
}

\section{RESUMO}

O estudo relacionou expectativas acadêmicas, motivação, habilidades sociais e adaptação acadêmica e verificou o impacto das três primeiras variáveis na adaptação à universidade. Participaram 320 estudantes de diferentes graduações. Empregou-se a Escala de Motivação, o Inventário de Habilidades Sociais, a Escala Expectativas Acadêmicas e o Questionário de Vivências Acadêmicas-reduzido. A coleta de dados ocorreu in loco e utilizou-se correlação de Pearson e regressão linear múltipla. Todos os procedimentos éticos foram cumpridos. Os resultados evidenciaram uma relação preditiva das variáveis no escore total da adaptação acadêmica. Entende-se que expectativas atendidas em termos de um ambiente de aprendizagem enriquecedor assim como o desenvolvimento de habilidades sociais promovem a adaptação acadêmica e o crescimento profissional e pessoal. O estudo mostrou que as expectativas permitem indicar os fatores determinantes do processo de adaptação ao Ensino Superior. Investigação prospectiva pode considerar dados sociodemográficos e o fato de o aluno ser de instituições públicas e privadas.

Palavras-chave: adaptação; habilidades sociais; motivação.

\section{Academic adaptation to the university: relationships among motivation, expectations and social skills}

\begin{abstract}
The study related academic expectations, motivation, social skills and academic adaptation and verified the impact of the first three variables on university adaptation. 320 students of different degrees participated. The Motivation Scale, the Social Skills Inventory, the Academic Expectations Scale and the Reduced Academic Experiences Questionnaire were used. Data collection took place in loco and Pearson correlation and multiple linear regression were used. All ethical procedures were followed. The results showed a predictive relationship of the variables in the total score of academic adaptation. It is understood that expectations met in terms of an enriching learning environment as well as the development of social skills promote academic adaptation and professional and personal growth. The study showed that expectations allow us to indicate the determining factors in the process of adapting to Higher Education. Prospective research can consider sociodemographic data and the fact that the student is from public and private institutions.

Keywords: adaptation; social skills; motivation.

\section{Adaptación académica a la universidad: relaciones entre motivación, expectativas y habilidades sociales \\ RESUMEN}

En el estudio se relacionó expectativas académicas, motivación, habilidades sociales y adaptación académica y se verificó el impacto de las tres primeras variables en la adaptación a la universidad. Participaron 320 estudiantes de distintas graduaciones. Se empleó la Escala de Motivación, el Inventario de Habilidades Sociales, la Escala Expectativas Académicas y el Cuestionario de Vivencias Académicas-reducido. La recolecta de datos sucedió in situ y se utilizó correlación de Pearson y regresión linear múltiple. Todos los procedimientos éticos fueron cumplidos. Los resultados evidenciaron una relación de predicción de las variables en el escore total de la adaptación académica. Se entendió que expectativas atendidas en términos de un ambiente de aprendizaje de buena calidad, así como el desarrollo de habilidades sociales promueven la adaptación académica y el crecimiento profesional y personal. El estudio apuntó que las expectativas permiten indicar los factores determinantes del proceso de adaptación a la Enseñanza Superior. Investigación prospectiva puede considerar datos sociodemográficos y el hecho de el alumno ser de instituciones públicas y privadas.

Palabras clave: adaptación; habilidades sociales; motivación.

\footnotetext{
${ }^{1}$ Universidade do Estado do Rio de Janeiro - UERJ - Rio de Janeiro - RJ - Brasil; adribenevides@gmail.com

${ }^{2}$ Universidade Salgado de Oliveira - UNIVERSO - Rio de Janeiro - RJ - Brasil; marcialauriapsi@outlook.com; hcpasseri@gmail.com; maiafalmeida@gmail.com; rnbarros@yahoo.com.br
} 


\section{INTRODUÇÃO}

A entrada para o Ensino Superior requer dos estudantes um envolvimento antes, durante e depois de seu acesso, pois vai exigir domínios e aprendizagens que os levarão a concretização de projetos de vida que envolvem a futura carreira. $O$ processo que antecede a entrada do estudante à universidade deveria incluir buscas de informações sobre as profissões, informações sobre as Instituições de Ensino Superior e seus programas, mas muitas vezes isso não acontece. Essa transição nem sempre é um momento fácil, pois compreende desafios e cobranças que são necessárias para que o estudante consiga enfrentar as mudanças próprias da idade, tomar decisões importantes e encarar as dificuldades que são pertinentes à chegada ao Ensino Superior (Almeida, Soares, \& Ferreira, 2002; Almeida, 2007; Almeida \& Cruz, 2010).

As vivências na educação superior também representam para muitos estudantes saber administrar de forma habilidosa novos padrões no relacionamento grupal. O aluno precisa lidar com as diferentes características de cada professor, com o afastamento da família e com a responsabilidade para gerir de forma autônoma seus recursos financeiros, podendo em muitos casos, encontrar-se despreparado para essa nova etapa (Almeida, 2007). Entretanto, tais demandas irão exigir estratégias (cognitivas e relacionais) que possibilitem ao estudante concluir as atividades e lidar com as diversidades impostas pelos novos vínculos, como gerir o tempo para as diferentes tarefas, construir e manter novos vínculos com pares e professores.

Dessa forma, compreende-se que as expectativas acadêmicas são como objetivos e desejos que envolvem os alunos, podendo influenciar o nível de empenho, de compromisso e de esforço, sendo um dos fatores relevantes no processo de adaptação à universidade (Vasconcelos, Almeida, \& Monteiro, 2009). Importante considerar que o aluno ao demonstrar baixas expectativas e pouco envolvimento acadêmico, diminui a probabilidade de obtenção de sucesso e de permanência à universidade, podendo abandonar o curso ou apresentar menor comprometimento (Almeida, Guisande, \& Paisana, 2012; Bisinoto, Rabelo, Marinho-Araújo, \& Fleith, 2016; Igue, Bariani, \& Milanesi, 2008).

Em estudo desenvolvido por Bisinoto et al. (2016) com o objetivo de compreender as expectativas que os alunos iniciantes apresentam ao ingressarem na universidade foram relacionadas, em ordem de importância, as seguintes dimensões: Formação Acadêmica de Qualidade, Perspectiva de Sucesso Profissional, Oportunidade de Internacionalização e Intercâmbio, Compromisso Social e Acadêmico, Ampliação das Relações Interpessoais e Preocupação com Autoimagem. Os autores relacionam as expectativas dos alunos com a Política de Acolhimento Acadêmico existente na univer- sidade que possibilita orientar o planejamento, as ações e os serviços, o trabalho em rede, assim como avaliar as iniciativas institucionais direcionadas à melhoria do ensino na universidade.

Face às diversidades de expectativas ao ingressar na universidade e diante das dificuldades encontradas na transição e na adaptação ao Ensino Superior, o estudante pode deparar-se com situações bem complexas, crescentes no grupo que compõe o novo espaço acadêmico e que não correspondem às expectativas iniciais (Almeida \& Cruz, 2010). Segundo os autores, observa-se uma diversidade de estudantes que iniciam o curso superior oriundos de diferentes origens socioeconômicas, com pouca tradição universitária familiar, conhecimentos prévios insuficientes para a aquisição de novas demandas educacionais, além de baixas capacidades cognitivas que podem dificultar o desempenho acadêmico do universitário. Compreende-se que, embora a heterogeneidade esteja cada vez mais presente, o acesso ao novo público estudantil permite que alunos que antes não tinham a possibilidade de cursar o Ensino Superior, possam estar bastante motivados para superar as dificuldades e alcançar um novo patamar profissional que antes não lhes era permitido.

Estudos sobre motivação (Almeida \& Cruz, 2010; Bzuneck, 2009; Ruiz, 2005), no campo educacional, têm trazido uma compreensão significativa em relação ao direcionamento e à energia na aprendizagem (Sobral, 2009); portanto, é possível supor que algumas dificuldades encontradas pelos alunos possam ser sobrepostas dado o grau de motivação encontrada. Lens, Matos e Vansteenkiste (2008, p. 17), conceituam a motivação como um "processo psicológico no qual interatuam as características de personalidade (por exemplo, motivos, razões, habilidades, interesses, expectativas, perspectiva de futuro) e as características ambientais percebidas". Ainda ressaltam que a motivação do estudante pode sofrer mudanças neles mesmos (aumentando sua percepção de autoeficácia, diminuindo ansiedades nas avaliações), bem como em seu ambiente de aprendizagem e na cultura escolar (o currículo, o ambiente da sala de aula, no qual o professor tem um papel relevante). Já Bergamini (1997) enfatiza a importância dos aspectos externos (do contexto) e internos (pessoais) como decisivos para a motivação. No ambiente universitário, a qualidade do investimento pessoal e a dedicação aos estudos têm efeito imediato na motivação (Ruiz, 2005; Santos, Mognon, Lima, \& Cunha, 2011), pois não basta que o estudante faça algum esforço, mas exige-se que encare tarefas desafiadoras, com persistência e profundidade, a fim de obter bons resultados na aprendizagem (Bzuneck, 2009). Segundo Almeida e Cruz (2010), alunos menos motivados e com dificuldades de encarar situações difíceis que são peculiares ao Ensino Superior, podem prejudicar sua adaptação e enfrentar maiores 
desafios em relação ao sucesso acadêmico.

Wibrowski, Matthews e Kitsantas (2016) realizaram estudo longitudinal sobre o impacto do Skills Learning Support Program (SLSP) sobre as crenças motivacionais em estudantes universitários ingressantes de primeira geração, no uso de estratégias autorreguladoras e na realização acadêmica. $O$ treinamento envolveu a preparação para os alunos enfrentarem os desafios sociais e acadêmicos, tais como seminários, cursos de aprimoramento na língua inglesa, até cursos sobre autopercepção e relacionamentos. Os resultados apontaram que houve mudanças positivas nas estratégias de aprendizagem, de gestão de recursos, autoeficácia, motivação intrínseca, valores das experiências acadêmicas e orientação de metas presentes nos pré e pós-testes. Além disso, os alunos envolvidos no treinamento apresentaram conquistas acadêmicas iguais ou superiores aos estudantes regularmente matriculados desde o primeiro ano.

Tendo considerado as expectativas e a motivação como construtos fundamentais para a boa adaptação acadêmica inicial, principalmente no primeiro ano do curso, é importante levar em conta que as habilidades relacionais devem perpetuar ao longo dos anos no Ensino Superior numa continuidade dessa integração e ajustamento a essa nova etapa da vida. Na trajetória do estudante, alguns fatores como o bom relacionamento com os professores e colegas de turma, o apoio e a qualidade das relações familiares podem contribuir ou implicar em sua adaptação acadêmica (Polydoro, 2000; Soares et al., 2014). Soares e Del Prette (2015) destacam que as habilidades sociais são facilitadoras do desempenho técnico e social do estudante. As habilidades sociais, segundo Del Prette e Del Prette (2001) são diferentes classes de comportamentos sociais do repertório de um indivíduo para lidar com os diferentes desafios dos vários contextos sociais. A universidade é um ambiente social, no qual o estudante promove ou inibe suas relações sociais entre pares, professores, funcionários e administradores. Quando promove uma interação satisfatória, aumenta a probabilidade de ter sucesso nas atividades acadêmicas, diminuindo as tensões. Portanto, as habilidades sociais têm sido associadas à adaptação acadêmica, numa perspectiva de facilitar ao universitário um maior envolvimento em suas atividades, alcançando com sucesso suas metas profissionais (Couto, Vandenberghe, Tavares, \& Silva, 2012).

Comedis (2014) investigou a relação entre as habilidades sociais de cooperação, empatia, autocontrole, assertividade e desempenho acadêmico de alunos ingressantes em sociologia. $O$ estudo mostrou que o autocontrole e as habilidades sociais no geral apontaram relação significativa com o desempenho acadêmico. 0 autor lembra que a generalização dos resultados está limitada aos alunos de primeiro ano e que é possível que as habilidades sociais tenham alta relação com o desempenho acadêmico no período de transição entre o Ensino Médio e o Superior. Já o estudo realizado por Mello e Soares (2014), as autoras investigaram a relação entre habilidades sociais e frustração em estudantes ingressantes e formandos de Medicina. A pesquisa mostrou que existem diferenças entre iniciantes e concluintes quanto às reações de frustração, habilidade social assertiva e empática no que se refere à perspectiva e sentimentos do outro e altruísmo. $O$ estudo revelou ainda que quanto mais os estudantes superam os desafios pertinentes à atuação como futuros médicos, assumindo as atribuições, mais competência social apresentam com os pacientes, o que conduziria a um melhor desempenho profissional.

As reais dificuldades enfrentadas pelos estudantes na transição e adaptação acadêmica podem ser compreendidas como uma série de desafios que encontram no campo motivacional, das habilidades sociais e das expectativas acadêmicas nas situações vivenciadas pelos calouros. Essas situações precisam ser experienciadas positivamente para que o aluno consiga diminuir a probabilidade de problemas futuros como o insucesso e abandono do curso. Portanto, este estudo tem como objetivos 1) relacionar os construtos expectativas acadêmicas, motivação, habilidades sociais e adaptação acadêmica e 2) verificar o impacto das três primeiras variáveis na adaptação à universidade.

\section{MÉTODO}

\section{Participantes}

Participaram do estudo 320 estudantes, pertencentes a instituições públicas $(\mathrm{N}=87)(27,19 \%)$ e privadas $(\mathrm{N}=233)(72,81 \%)$ de diferentes graduações, de ambos os sexos sendo $63,1 \%$ mulheres e $36,9 \%$ homens. Os voluntários eram do primeiro $(\mathrm{N}=222)(69,38 \%)$ e segundo períodos $(\mathrm{N}=98)(30,63 \%)$. As idades variaram entre 17 a 45 anos $(M=22,47 ; D P=4,73)$. Quanto a classe social, $29(9,06 \%)$ pertenciam à $A, 40(12,50 \%)$ à $B 1,93(29,06 \%)$ à $B 2,100(31,25 \%)$ à $C 1,42(13,13 \%)$ $C 2,15(4,69 \%)$ à DE e um $(0,31 \%)$ não informou.

\section{Instrumentos}

Escla de Motivação Acadêmica (EMA) (Sobral, 2003). É constituída de 28 itens subdivididos em sete subescalas: três correspondem a tipos de motivação intrínseca; outras três integram tipos de motivação extrínseca e, a última, representa a desmotivação ou ausência de motivação. As respostas devem ser escolhidas em uma escala tipo Likert que varia de (1- nenhuma correspondência) a (7- total correspondência).

Inventário de Habilidades Sociais (IHS) (Del Prette \& Del Prette, 2001) é um instrumento de autorrelato para avaliação de habilidades sociais. Compõe-se de 38 itens, descrevendo uma situação de relação interpes- 
soal e uma demanda de habilidade para reagir àquela situação. O respondente deve estimar a frequência com que reage da forma sugerida em cada item e estimar a frequência de sua resposta em escala tipo Likert, com cinco pontos, variando de zero (nunca ou raramente) a 4 (sempre ou quase sempre).

Escala Expectativas Acadêmicas de Estudantes Ingressantes na Educação Superior (Marinho-Araújo, Fleith, Almeida, Bisinoto, \& Rabelo, 2015) adaptada do Questionário de Percepções Acadêmicas, versão A Expectativas (QPA-E) (Almeida, Costa, Alves, Gonçalves, \& Araújo, 2012). A escala contém 61 itens e as respostas devem ser escolhidas em um espectro de seis pontos, considerando um contínuo entre pouco e muito.

Questionário de Vivências Acadêmicas-reduzido (QVA-r) (Almeida et al., 2002). É constituído de 55 itens em uma escala do tipo Likert de cinco pontos, indo de 1 (nada a ver comigo) a 5 (tudo a ver comigo). 0 instrumento abrange cinco dimensões: Pessoal, Interpessoal, Vocacional, Estudo-aprendizagem e Institucional e Alpha de Cronbach variando de 0,72 a 0,87 nas subescalas.

\section{Procedimentos de Coleta de dados}

Inicialmente foi realizado um contato com as coordenações de cursos das instituições para apresentação do estudo e convite aos alunos para a participação. Os instrumentos foram aplicados de forma presencial e coletiva, em sala de aula e na seguinte ordem: Escala de Motivação Acadêmica, Escala de Expectativa Acadêmica no Ensino Superior, Inventário de Habilidades Sociais, Questionário de Vivências Acadêmicas-reduzido e Questionário Sociodemográfico.

\section{Procedimentos de Análise de dados}

Para relacionar os construtos dois a dois foi utilizado o teste de correlação de Pearson e para avaliar o impacto das variáveis independentes expectativas acadêmicas, motivação e habilidades sociais na variável dependente adaptação acadêmica foi utilizado o teste de regressão linear múltipla.

\section{Procedimentos éticos}

Todos os participantes assinaram o Termo de Consentimento Livre e Esclarecido, foram informados do objetivo do estudo, do caráter sigiloso dos dados e da possibilidade de interromper a qualquer momento a participação. Na coleta de dados, um e-mail foi disponibilizado para contato permanente. O estudo foi aprovado pelo Comitê de Ética e Pesquisa da Universidade.

\section{RESULTADOS}

Foram encontradas relações estatisticamente significativas entre os escores totais do QVA-r (Questionário de Vivências Acadêmicas-reduzido) com os escores totais da Escala de Expectativa Acadêmica
(EEA) e da Escala de Motivação Acadêmica (EMA). Porém, o escore total do QVA-r não se relaciona com o escore total do IHS (Inventário de Habilidades Sociais). O escore total do IHS não apresenta correlação significativa com os escores da EEA e EMA. A correlação mais forte encontrada foi entre o escore total da EEA e o escore total da EMA.

A Tabela 1 apresenta o impacto das variáveis de motivação, expectativas acadêmicas e habilidades sociais no escore total da adaptação acadêmica. Os resultados da análise de regressão linear múltipla (RLM) mostram haver uma predição das variáveis no escore total da adaptação acadêmica $[F(18,274)=7,014, p<0,001]$, explicando $27,0 \%$ dos escores $\left(R^{2}\right)$. Em relação à EEA, o fator Formação Acadêmica de Qualidade apresentou o impacto negativo nos escores totais do QVA-r $(\beta=$ $-0,235, p<0,05)$. Já o fator Desenvolvimento de Competências Transversais apresentou impacto positivo ( $\beta$ $=0,230, p<0,05)$. Ambos os resultados indicam que quanto mais o estudante espera da instituição em termos, por exemplo, de um ambiente de aprendizagem desafiador, uma estrutura física que possibilite a pesquisa e extensão, assim como atividades tanto curriculares como extracurriculares, mais fácil torna-se a adaptação do estudante à universidade. Em relação à EMA, apenas o fator Motivação Intrínseca para o Saber $(\beta=0,243, p$ $<0,01)$ apresentou impacto positivo nos escores totais do QVA-r. Tal resultado aponta e reforça o fato que o estudante busca o aprendizado, exigindo que as atividades proporcionadas na universidade tenham relação estreita com o processo de ensino-aprendizagem. Por fim, em relação ao IHS, o fator Enfrentamento e Autoafirmação com Risco impactou positivamente os escores totais do QVA-r $(\beta=0,152, p<0,001)$, enquanto o fator Conversação e Desenvoltura Social impactou negativamente $(\beta=-0,239, p<0,001)$. O resultado apresentado pode indicar que as relações construídas no processo de adaptação ao Ensino Superior precisam ser enfatizadas tanto nos aspectos cognitivos e acadêmicos, como nas relações interpessoais. O aluno precisa desenvolver habilidades para lidar com os diferentes atores da comunidade universitária, defendendo ideias, sustentando opiniões, recebendo e fazendo críticas em benefício do próprio crescimento profissional e pessoal. De todas as variáveis significativas, a que apresentou maior poder de predição foi a Motivação Intrínseca para o Saber da $\operatorname{EMA}(\beta=0,243, p<0,001)$. Por fim, estimou-se pela RLM o impacto das variáveis motivação, expectativas acadêmicas e habilidades sociais na adaptação acadêmica. Os resultados da RLM demonstram haver uma predição das variáveis na adaptação acadêmica $[F(3,315)=25,531$, $p<0,001$ ], explicando $18,3 \%$ dos escores de adaptação acadêmica (R2). A EEA e EMA impactaram significativamente nos escores de adaptação acadêmica, mas não houve influência da IHS. De todas as variáveis, a que 
Tabela 1. Impacto das Variáveis de Motivação, Expectativas Acadêmicas e Habilidades Sociais no Escore Total da Adaptação Acadêmica

\begin{tabular}{|c|c|c|c|c|c|c|}
\hline \multirow{2}{*}{ QVA-r Total } & \multicolumn{2}{|c|}{$\begin{array}{c}\text { Coeficientes } \\
\text { não-padronizados }\end{array}$} & \multirow{2}{*}{$\frac{\begin{array}{c}\text { Coeficientes } \\
\text { padronizados }\end{array}}{\text { Beta }}$} & \multirow[t]{2}{*}{$\mathbf{t}$} & \multirow{2}{*}{ Sig. } & \multirow[t]{2}{*}{$\mathbf{R}^{2}$} \\
\hline & B & Erro padrão & & & & \\
\hline (Constant) & 2,453 & 0,219 & & 11,223 & 0,000 & \\
\hline EEA_Form_Acad & $-0,165$ & 0,058 & $-0,281$ & $-2,844$ & 0,005 & \\
\hline EEA_Comp_Soc_Acad & 0,091 & 0,036 & 0,186 & 2,538 & 0,012 & \\
\hline EEA_Amp_Rel_Soc & 0,059 & 0,036 & 0,117 & 1,639 & 0,102 & \\
\hline EEA_Op_Intern_Interc & $-0,004$ & 0,022 & $-0,012$ & $-0,197$ & 0,844 & \\
\hline EEA_Persp_Suc_Prof & 0,033 & 0,035 & 0,066 & 0,957 & 0,339 & \\
\hline EEA_Preocup_Al & 0,040 & 0,024 & 0,108 & 1,642 & 0,102 & \\
\hline EEA_Des_Compet_Transv & 0,104 & 0,044 & 0,201 & 2,353 & 0,019 & \\
\hline EMA_MIC & $-0,016$ & 0,026 & $-0,045$ & $-0,592$ & 0,555 & \\
\hline EMA_MIR & 0,077 & 0,025 & 0,253 & 3,113 & 0,002 & \\
\hline EMA_MIVE & 0,021 & 0,020 & 0,079 & 1,078 & 0,282 & 0,200 \\
\hline EMA_ME_ID & 0,027 & 0,029 & 0,071 & 0,932 & 0,352 & \\
\hline EMA_ME_INT & $-0,015$ & 0,020 & $-0,056$ & $-0,757$ & 0,450 & \\
\hline EMA_ME_CE & $-0,026$ & 0,020 & $-0,084$ & $-1,300$ & 0,194 & \\
\hline EMA_DESM & $-0,010$ & 0,012 & $-0,047$ & $-0,833$ & 0,406 & \\
\hline HIS_EAAR & 0,077 & 0,033 & 0,131 & 2,341 & 0,020 & \\
\hline HIS_AAESP & $-0,004$ & 0,034 & $-0,007$ & $-0,123$ & 0,903 & \\
\hline HIS_CDS & $-0,096$ & 0,029 & $-0,194$ & $-3,280$ & 0,001 & \\
\hline HIS_AEDSN & 0,005 & 0,029 & 0,011 & 0,182 & 0,856 & \\
\hline HIS_ACA & 0,012 & 0,029 & 0,021 & 0,402 & 0,688 & \\
\hline \multicolumn{7}{|l|}{ QVA-r-Total } \\
\hline Constante & 2,29 & 0,18 & & 12,55 & 0,00 & \multirow{4}{*}{0,183} \\
\hline EEA_Total & 0,170 & 0,03 & 0,28 & 5,01 & 0,00 & \\
\hline EMA_Total & 0,10 & 0,03 & 0,23 & 3,85 & 0,00 & \\
\hline HIS_Total & $-0,07$ & 0,05 & $-0,08$ & $-1,47$ & 0,14 & \\
\hline
\end{tabular}

Fonte: Elaboração própria.

impactou mais fortemente foi a $\operatorname{EEA}(\beta=0,28, p<0,001)$.

\section{DISCUSSÃO}

A pesquisa teve como objetivo relacionar os construtos expectativas acadêmicas, motivação, habilidades sociais e adaptação acadêmica e verificar o impacto dos três primeiros construtos na adaptação à universidade. No que diz respeito ao primeiro objetivo, os resultados apontaram que existe relação significativa entre o escore total do QVA-r (a soma total dos valores obtidos) e o escore total da EEA (a soma total de todos os valores obtidos). Sendo assim, as expectativas dos estudantes permitem evidenciar fatores determinantes ao processo de adaptação ao Ensino Superior. Os resultados obtidos por lgue et al. (2008) corroboraram com o obtido nesse estudo. Os autores descreveram as vivências acadêmicas de estudantes universitários com objetivo de verificar se essas se modificavam de acordo com o ano de estudo. Analisaram se as expectativas e as vivências dos alunos do 10 e do 50 apresentavam diferenças. Encontraram diferenças significativas na dimensão Institucional das vivências dos iniciantes. Os alunos de 50 ano apresentaram mais expectativas que os iniciantes com $79,7 \%$ dos estudantes na categoria "altas" ou "muito altas". Esse dado pode indicar que a satisfação com as experiências vividas pode contribuir para o processo adaptativo e para a certeza quanto a escolha da carreira, segundo os autores.

Os resultados encontrados no presente estudo corroboram que as vivências na universidade se relacionam com os tipos e níveis de expectativas dos estudantes e quando essas expectativas são mais elevadas se associam a melhores índices de adaptação acadêmica. Os resultados do presente estudo apontam ainda que há 
relações estatisticamente significativas entre os escores totais do QVA-r com os escores totais da EMA, porém há ausência de correlação do escore total do QVA-r com o escore total do IHS. Santos et al. (2011) pesquisaram as relações entre a vida acadêmica e a motivação para aprendizagem com 239 universitários. Os resultados encontrados apontaram que existe relação positiva e significativa entre a meta aprendizagem e as habilidades do estudante. Essas habilidades referem-se ao entendimento de suas competências cognitivas, tais como expressão, compreensão, resolução de problemas e concentração. A associação negativa da meta de aprendizagem com o fator envolvimento em atividades não obrigatórias e com o fator condições para o estudo e desempenho acadêmico aponta no sentido de que toda e qualquer tarefa que não esteja relacionada ao formal exigido para o curso pode ocupar um tempo importante dos estudos, embora contribuam para uma maior integração, persistência e esforço para se integrar (Santos, Alcará, \& Zenorini, 2013).

Embora o presente estudo tenha apontado a ausência de correlação do escore total do QVA-r com o escore total do IHS, a pesquisa de Soares et al. (2014) aponta que diante de um maior envolvimento social do aluno, suas chances serão aumentadas para alcançar relações interpessoais satisfatórias na universidade, o que significa que um bom relacionamento interpessoal tende a favorecer o processo de adaptação e o sucesso acadêmico (Almeida et al., 2002; Polydoro, 2000). Conforme os autores, o universitário enfrenta um período adaptativo que demanda uma série de competências cognitivas e comportamentais para atingir suas metas. As variadas interações com grupos de características diferenciadas nos aspectos acadêmicos podem levar os estudantes a não saber como lidar com essas demandas específicas. As deficiências neste processo interativo das ações de estudo, cooperação e realização de trabalhos pode dificultar a adaptação no contexto universitário. A melhor ou pior vivência nesse ambiente tem em seu bojo influências das habilidades sociais e sua dinâmica é consequência dos comportamentos sociais (Soares et al., 2014).

A correlação encontrada entre o escore total da EEA com o escore total da EMA pode ser compreendida pelo fato de que uma associação positiva entre duas varáveis psicológicas no espectro do relacionamento interpessoal e curricular sinalizam que as expectativas acadêmicas ligadas ao envolvimento social, vocacional e curricular se associam a adaptação do aluno ao Ensino Superior (Soares et al., 2014). A revisão de literatura realizada por Ruiz (2005) identificou variáveis que influenciam a motivação para aprender de estudantes universitários. Foi utilizado o modelo de Pintrich, que incorpora quatro componentes principais: contexto sociocultural, fatores relacionados ao ambiente de sala de aula, fatores internos e comportamento motivado. Dessa forma, espera-se que para o aluno concluir com êxito sua formação é preciso que ele saiba utilizar de forma autodeterminada, autorregulada, dirigida e ativa as estratégias para conduzir tanto sua motivação quanto o seu comportamento e a sua aprendizagem. A vivência em cada situação pode servir de base na busca pelo aperfeiçoamento de suas habilidades para alcançar a construção do conhecimento dentro dessa diversidade (Leal, Miranda, \& Carmo, 2013).

A motivação e as expectativas acadêmicas impactam na adaptação acadêmica, tendo papéis relevantes na trajetória do estudante à universidade (Marinho-Araújo et al., 2015). A motivação pode ser compreendida como um construto que apresenta determinantes tanto ambientais como pessoais, impulsionando o sujeito a agir e ir adiante em direção aos seus objetivos (Bergamini, 1997). Ao cumprir trabalhos e atividades explorando e pesquisando conteúdos novos, o estudante pode estar intrinsicamente motivado a aprender e a explorar o que ainda desconhece. Assim, a motivação pode propiciar o conhecimento de algo novo relacionado à aprendizagem, dispondo ao estudante aprofundar seu nível de participação e conhecimento para a superação acadêmica (Falcão \& da Rosa, 2008). No que diz respeito às expectativas acadêmicas, Marinho-Araújo et al. (2015) trazem a ideia de que estas variáveis terão influências na permanência dos estudantes no curso, na medida em que os diferentes desafios percebidos determinam ou não o processo de integração ao Ensino Superior. Os autores apontam que a chegada à universidade reflete importantes sentimentos traduzidos como crescimento e realização profissional, a qualidade do curso e das instituições, as propostas curriculares, as altas expectativas com as aulas e com os professores. Pode ser que as expectativas acadêmicas que impactaram fortemente nesse estudo sejam, segundo Polydoro (2000), marcadas pelo entusiasmo do novo ambiente tão desejado e idealizado como um espaço que muda a vida de pessoas e suas condições profissionais futuras.

Alguns estudos (Igue et al., 2008; Vasconcelos et al., 2009) são consensuais em mostrar que as expectativas dos alunos têm relação com a adaptação, integração e satisfação do estudante no Ensino Superior, contribuindo para a permanência do estudante na IES. A estimativa dos dados aponta para o fato que é a partir das expectativas acadêmicas que o estudante supostamente pode compreender o ambiente de aprendizagem e as competências necessárias para o sucesso na universidade. Supõe-se que expectativas iniciais dos alunos, no que concerne ao que esperam encontrar na universidade são revestidas de concepções agradáveis e afáveis sobre o novo ambiente escolar.

Os resultados mostram ainda que as expectativas dos estudantes estão também relacionadas às relações 
interpessoais e amizades desenvolvidas na universidade, por esse motivo a preocupação com a autoimagem. Em outros termos, pode-se interpretar que o estudante está motivado a regular a expressão de comportamentos controlando a autoapresentação no intuito de adequar-se ao contexto (Snyder, 1974). Por outro lado, a expectativa de Formação Acadêmica com qualidade indica que o estudante além de almejar a certificação espera também alcançar sucesso profissional, remuneração, reconhecimento, isto é, status social que a futura carreira pode proporcionar (Moreno \& Soares, 2014).

Finalmente, apesar das competências transversais serem reconhecidas como um dos objetivos da Educação Superior (Castro \& Almeida, 2016), os alunos parecerem não estar atentos ou mesmo motivados a desenvolverem capacidades que não apresentem uma estreita relação com a graduação, mostrando supostamente um desconhecimento de diferentes aspectos que envolvem a vida acadêmica, tais como o desenvolvimento psicossocial, os objetivos gerais do curso e específicos das disciplinas e mesmo as exigências vinculadas ao mercado de trabalho.

\section{CONSIDERAÇÕES FINAIS}

O objetivo do estudo foi relacionar os construtos expectativas acadêmicas, motivação, habilidades sociais e adaptação acadêmica e verificar o impacto dos três primeiros na adaptação à universidade. Assim, ao ingressar no Ensino Superior o estudante inicia uma nova etapa de vida cercada de diferentes expectativas, algumas delas fantasiosas, sobre a modalidade de ensino e a futura carreira. Conhecer as expectativas iniciais dos alunos e como essas se modificam ao longo dos anos de graduação, considerando, por exemplo, as vivências de estágio, a aproximação do término do curso e a inserção no mercado de trabalho, seria de relevância para integração do discente às instituições de ensino. Entender as expectativas iniciais trazidas pelos alunos e como elas se transformam facilitaria a elaboração e promoção de ações que despertem no estudante a identificação de suas expectativas, favorecendo a adaptação à universidade. Ressalta-se ainda que o estudo apontou para o fato de que as expectativas mais realistas se relacionam com uma melhor adaptação acadêmica.

Outrossim, o processo de aprendizagem envolve elementos motivacionais tanto internos como externos exigindo um ambiente pedagógico desafiador para o aluno. Dessa forma, as atividades curriculares e extracurriculares devem proporcionar efetivamente a aquisição de conhecimento, promovendo, inclusive, o compromisso do aluno com seu processo de ensino e aprendizagem e seu envolvimento social. O investimento institucional em infraestrutura, como bibliotecas e laboratórios e na qualidade das ações pedagógicas muito reconheceria a importância dos determinantes externos e internos que envolvem os processos motivacionais.

Ademais, a adaptação acadêmica abrange as relações interpessoais e um estudante socialmente competente consegue administrar e construir vínculos com pares, docentes e demais membros da comunidade universitária. O estudo indicou que estar engajado na universidade tanto com as pessoas como com o currículo, sinaliza que as expectativas acadêmicas relacionadas ao envolvimento social, da carreira e com disciplinas da graduação influenciam a adaptação do aluno ao Ensino Superior. Afinal, a universidade não forma apenas futuros profissionais, mas sujeitos preparados para lidar com os desafios da vida.

Em termos de limitações, o estudo poderia ter contado com uma amostra de estudantes de outros estados da federação, além de pesquisar o comportamento das variáveis considerando dados demográficos e o fato de os alunos serem advindos de instituições públicas e privadas. Estudos prospectivos poderiam focar nessa comparação e estabelecer relação das variáveis com o sucesso na carreira profissional.

\section{REFERÊNCIAS}

Almeida, L. S. (2007). Transição, adaptação académica e êxito escolar no ensino superior. Revista Galego-Portuguesa de Psicoloxía e Educación, 15(2), 203-215.

Almeida, L.; Costa, A. R.; Alves, F.; Gonçalves, P.; Araújo, A. M. (2012). Expectativas acadêmicas dos alunos do ensino superior: construção e validação de uma escala de avaliação. Psicologia, Educação e Cultura, 16(1), 70-85.

Almeida, L. S.; Cruz, J. F. A. (2010). Transição e adaptação académica: reflexões em torno dos alunos do 10 ano da Universidade do Minho. In: Silva, J. L. et. al. (Eds.), "Ensino superior em mudança: tensões e possibilidades: actas do Congresso Ibérico, Braga, Portugal, 2010" (pp. 429-440). Braga: CIEd. ISBN 978-972-8746-80-3.

Almeida, L. S.; Guisande, M.; Paisana, J. (2012). Extra-curricular involvement, academic adjustment and achievement in higher education: A study of Portuguese students. Anales de Psicologia, 28, 860-865.

Almeida, L. S; Soares, A. P.; Ferreira, J. A. G. (2002). Questionário de Vivências Acadêmicas (QVA-r): Avaliação do ajustamento dos estudantes universitários. Avaliação Psicológica, 1(2), 81-93.

Bergamini, C. W. (1997). A motivação nas organizações (4ạ ed). São Paulo: Atlas.

Bisinoto, C.; Rabelo, M. L.; Marinho-Araújo, C.; Fleith, D. S. (2016). Expectativas Acadêmicas dos Ingressantes da Universidade de Brasília: indicadores para uma política de acolhimento. In: Almeida, L. S.; Castro, R. V. (Eds.), Ser estudantes no Ensino Superior: o caso dos estudantes do 1‥ Ano (pp. 15-31). Braga: Universidade do Minho.

Bzuneck, J. A. (2009). A Motivação do Aluno: Aspectos Introdutórios. In: Boruchovith, E.; Bzuneck, J. A. (Eds.), A Motivação do Aluno - Contribuições da Psicologia 
Contemporânea (pp. 9-36). Petrópolis: Editora Vozes.

Castro, R. V.; Almeida L. S. (2016). Ser estudante no ensino superior: Observatório dos percursos académicos dos estudantes da Universidade do Minho. In: Almeida, L. S.; Castro, R. V. (Eds.), Ser estudante no Ensino Superior: $O$ caso dos estudantes do 1 o ano. Portugal (pp. 15-31). Braga: Universidade do Minho.

Comedis, E. J. (2014). The Role of Social Skills in the Academic Performance of De La Salle Araneta University Freshmen Students: Creating a Culture. Research Congress 2014. Philippines: De La Salle University. Recuperado de https://xsite.dlsu.edu.ph/conferences/dlsu_research_ congress/2014/_pdf/proceedings/LCCS-I-002-FT.pdf

Couto, G.; Vandenberghe, L.; Tavares, W. M.; Silva, R. L. F. C. (2012). Interações e habilidades sociais entre universitários: um estudo correlacional. Estudos de Psicologia, 29, 667677.

Del Prette, Z. A. P.; Del Prette, A. (2001). Inventário de Habilidades Sociais (IHS-Del-Prette): Manual de aplicação, apuração e interpretação. São Paulo: Casa do Psicólogo.

Falcão, D. F.; Rosa, V. V. da. (2008). Um estudo sobre a motivação dos universitários do curso de administração: uma contribuição para gestão acadêmica no âmbito público e privado. Anais do XXXII Encontro da Anpad. Rio de Janeiro - RJ.

Igue, E. A.; Bariani, I. C.; Milanesi, P. V. (2008). Vivência acadêmica e expectativas de universitários ingressantes e concluintes. PsicoUSF, 13(2), 155-164.

Leal, E. A.; Miranda, G. J.; Carmo, C. R. S. (2013). Teoria da Autodeterminação: uma análise da motivação dos estudantes do curso de ciências contábeis. Revista Contabilidade e Finanças, 24(62), 162-173.

Lens, W.; Matos, L.; Vansteenkiste, M. (2008). Professores como fontes de motivação dos alunos: o quê e o porquê da aprendizagem do aluno. Educação, 31(1), 17-20.

Marinho-Araújo, C. M.; Fleith, D. D. S.; Almeida, L. S.; Bisinoto, C.; Rabelo, M. L. (2015). Adaptação da Escala Expectativas Acadêmicas de Estudantes Ingressantes na Educação Superior. Avaliação Psicológica, 14(1), 133-141. DOI: 10.15689/ap.2015.1401.15

Mello, T. V. S.; Soares, A. B. (2014). Habilidades sociales y frustración en estudiantes de Medicina. Ciencias Psicológicas, 8(2), 163-172.

Moreno, P. F.; Soares, A. B. (2014). O que vai acontecer quando eu estiver na universidade? Expectativas de jovens estudantes brasileiros. Aletheia, 45, pp.114-127.
Polydoro, S. A. J. (2000). O trancamento de matrícula na trajetória acadêmica do universitário: Condições de saída e de retorno à instituição (Tese de Doutorado). Universidade Estadual de Campinas. Recuperado de http://repositorio. unicamp.br/jspui/handle/REPOSIP/253539

Ruiz, V. M. (2005). Aprendizagem em universitários: variáveis motivacionais (Tese de doutorado). Pontifícia Universidade Católica de Campinas. Recuperado de http://tede. bibliotecadigital.puc-campinas.edu.br:8080/jspui/handle/ tede/368

Santos, A. A. A.; Alcará, A. R.; Zenorini, R. P. C. (2013). Estudos psicométricos da escala de motivação para a aprendizagem de universitários. Fractal, 25(3), 531-546.

Santos, A. A. A.; Mognon, J. F.; Lima, T. H.; Cunha, N. B. (2011). A relação entre vida acadêmica e a motivação para aprender em universitários. Psicologia Escolar e Educacional, 15(2), 283-290.

Snyder, M. (1974). Self-monitoring of expressive behavior. Journal of Personality and Social Psychology, 30(4), 526-537.

Soares, A. B.; Francischetto, V.; Dutra, B. M.; de Miranda, J. M.; Nogueira, C. C. C.; Leme, V. R.; Araújo, A. M.; Almeida, L. S. (2014). O impacto das expectativas na adaptação acadêmica dos estudantes no Ensino Superior. Psico-USF, 19(1), 49-60.

Soares, A. B.; Dell Prette, Z. A. P. (2015). Habilidades sociais e adaptação à universidade: Convergências e divergências dos construtos. Análise Psicológica, 33(2), 139-151. DOI: 10.14417/ap.911

Sobral, D. T. (2003). Motivação do aprendiz de medicina: uso da Escala de Motivação Acadêmica. Psicologia: Teoria e Pesquisa, 19(1), 25-31.

Sobral, D. T. (2009). Padrão de motivação e desfechos de progresso acadêmico: estudo longitudinal com estudantes de medicina. ETD - Educação Temática Digital, 10, 228-248.

Vasconcelos, R. M.; Almeida, L. S.; Monteiro, S. (2009). O insucesso e o abandono acadêmico na universidade: Uma análise sobre os cursos de engenharia. Sixth International Conference on Engineering and Computer Education. Buenos Aires: Argentina. Recuperado de http://hdl.handle. net/1822/9412

Wibrowski, C.R.; Matthews, W. K. Kitsantas, A. (2016). The role of a skills learning support program on first-generation college students' self-regulation, motivation, and academic achievement: A longitudinal study. Journal of College Student Retention: Research, Theory \& Practice, 0(0), 1-16. 